\title{
THE SPACE OF ENTIRE FUNCTIONS OF TWO VARIABLES AS A METRIC SPACE
}

\author{
W. C. SISARCICK \\ Department of Mathematics \\ Marshall University \\ Huntington, West Virginia 25701 \\ (Received October 17, 1977)
}

Section 1. Introduction.

Let $\Gamma^{2}$ denote the space of entire functions of two variables. $\infty$ If $f(z, w) \in \Gamma^{2}, f(z, w)=\sum_{m, n=0} a_{m, n} z^{m} w^{n}$, the series converging absolutely for all $(z, w)$ and uniformly in every bicylinder centered at $(0,0),[2]$. Here, a metric is defined on $\Gamma^{2}$ and three classes of linear functionals on $\Gamma^{2}$ are characterized. We use the following notation. 
(1)

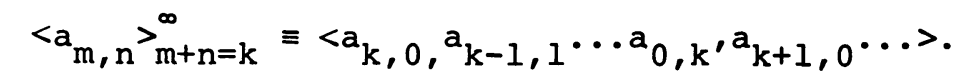

(2)

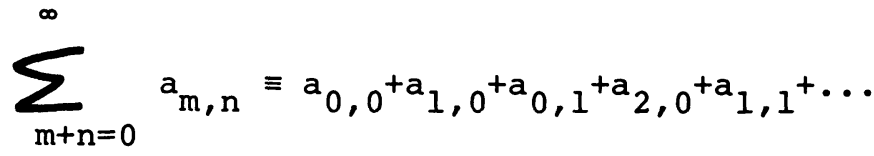

$$
\equiv \lim _{N \rightarrow \infty} \sum_{m+n=0}^{N} a_{m, n}
$$

Definition 1.1. The sequence $<a_{m, n}>_{m+n=k}^{\infty}$ is said to have limit a as $m+n \rightarrow \infty$, written $\lim _{m+n \rightarrow \infty} \bar{a}_{m, n}=a$, if and only if for any $\epsilon>0$, there exists an $N=N(\varepsilon) \geq 0$ such that $\left|a_{m, n}-a\right|<\varepsilon$ if $\mathrm{m}+\mathrm{n}>\mathrm{N}$.

Lemma 1.2. If $f(z, w)=\sum_{m, n=0}^{\infty} a_{m, n} z^{m} w^{n} \in \Gamma^{2}$, then for each $(z, w)$, the sequence $<a_{m, n} z^{m} w^{n}>_{m+n=0}^{\infty}$ is such that $\lim _{n \rightarrow \infty} a_{m, n} z^{m} w^{n}=0$.

Proof. Given $(z, w)$, let $s_{N}=\sum_{j+k=0}^{N}\left|a_{j, k} z^{j}{ }_{w}^{k}\right| \cdot \operatorname{since} \underset{\infty \rightarrow \infty}{l i m} s_{N}$ exists, $0=\lim _{N \rightarrow \infty} s_{N}-\lim _{N \rightarrow \infty} s_{N-1}=\lim _{N \rightarrow \infty}\left(s_{N}-S_{N-1}\right)=\lim _{N \rightarrow \infty} \sum_{j+k=N}\left|a_{j, k} z^{j}{ }_{w}^{k}\right|$. Hence given $\epsilon>0$, there exists an $M=M(\epsilon)$ such that if $N>M$, $\left|a_{N-j, j} z^{N-j} w^{j}\right|<\epsilon$ for each $j, 0 \leq j \leq N$. Let $m+n=N$. Then $0 \leq n \leq N$ and $\left|a_{m, n} z^{m} w^{n}\right|<\epsilon$. Therefore given $\epsilon>0$, there exists an $M=M(\epsilon)$ such that $m+n>M \Rightarrow\left|a_{m, n} z^{m} w^{n}\right|<\epsilon$. Hence $\lim _{m+n \rightarrow \infty} a_{m, n} z^{m} w^{n}=0$.

Lemma 1.3. A necessary and sufficient condition that $\sum_{m, n=0}^{\infty} a_{m, n} z^{m} w^{n}$ 
is an entire function is that for the sequence $\left\langle\left|a_{m, n}\right|^{1 / m+n}\right\rangle_{m+n=1}$, one has $\lim _{m+n \rightarrow \infty}\left|a_{m, n}\right|^{1 / m+n}=0$.

Proof. Let $\sum_{m, n=0}^{\infty} a_{m, n^{2}} z^{m} \in \Gamma^{2}$ and $T=\prod_{m+n \rightarrow \infty}\left|a_{m, n}\right|^{1 / m+n}$.

If $T>0$, choose $(z, w)$ such that $|z| \geq|w|>1 / T . \quad(1 / T=0$ if

$T=\infty)$. Then choose $p$ such that $|w|>p>1 / T$. Then

$\left|\frac{1}{z}\right| \leq\left|\frac{1}{w}\right|<\frac{1}{p}<T$. By definition of $T$, there exists a sequence $<\left(m_{k}, n_{k}\right)>^{\infty} k=1$ such that $<m_{k}+n_{k}>_{k=1}^{\infty}$ increases monotonically to $\infty$ and $\left|a_{m_{k}, n_{k}}\right|^{\frac{1}{m_{k}+n_{k}}}>1 / p$ for all k. Hence $\mid a_{m_{k}, n_{k}}{ }^{m_{k}{ }_{w}{ }^{n_{k}} \mid>}$ $\left(\frac{z}{p}\right)^{m_{k}}\left(\frac{w}{p}\right)^{n_{k}}>1$. This contradicts Lemma 1.2. Therefore $T=0$. Hence $\lim _{m+n \rightarrow \infty}\left|a_{m, n}\right|^{1 / m+n}=0$. Conversely, let $\sum_{m, n=0}^{\infty} a_{m, n} z^{m} w^{n}$ be a series such that for the sequence $<\left|a_{m, n}\right|^{1 / m+n}>_{m+n=1}^{\infty}$, one has $\lim _{m+n \rightarrow \infty}\left|a_{m, n}\right|^{1 / m+n}=0$. To show this series is an entire function, it sufficies to show [2] the series converges for each $(z, w)$. Consider $(z, w)$ fixed. Choose $\mathrm{p}$ such that $|\mathrm{z}|<\mathrm{p}$ and $|\mathrm{w}|<\mathrm{p}$. Let $\mathrm{N}$ be such that $\mathrm{m}+\mathrm{n}$ $>N \Rightarrow\left|a_{m, n}\right|^{1 / m+n}<1 / p$. Then for $m+n>N,\left|a_{m, n} z^{m} w^{n}\right|<$ $\left(\left|\frac{z}{p}\right|\right) m\left(\sum_{\infty}^{|w|}\right)^{n}, \sum_{m+n=N+1}\left|a_{m, n^{2}} z^{m}\right| \leq \sum_{m+n=N+1}\left(\frac{|z|}{p}\right)^{m}\left(\frac{w \mid}{p}\right)^{n}<\infty$. Therefore $\sum_{m+n=0}\left|a_{m, n} z^{m} w^{n}\right|<\infty$. 
Let $s_{p, q}=\sum_{m=0}^{p} \sum_{n=0}^{q} a_{m, n} z^{m} w^{n}$. To show the series converges, it sufficies to show [1] that given $\epsilon>0$, there exists an $N=N(\epsilon)$ such that $\left|s_{p, q}-s_{m, n}\right|<\epsilon$ if $p>m>N$ and $q>n>N$. Since $\sum_{m+n=0}^{\infty}\left|a_{m, n} z^{m} w^{n}\right|<\infty$, given $\epsilon>0$, there exists an $M=M(\epsilon)$ such that $N>\max \{M, 1\} \Rightarrow \sum_{j+k=N+1}^{\infty} a_{j, k} z^{j} w^{k}<\varepsilon$. Choose such an $N$.

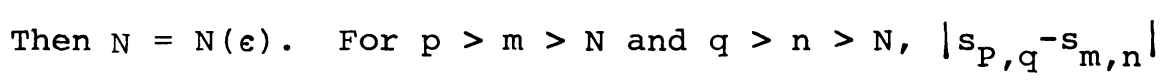
$=\left|\sum_{j=0}^{p} \sum_{k=0}^{q} a_{j, k} z^{j} w^{k}-\sum_{j=0}^{m} \sum_{k=0}^{n} a_{j, k} z^{j}{ }^{k}\right| \leq \sum_{j+k=m+n}^{\infty}\left|a_{j, k} z^{j} w^{k}\right|$ $\leq \sum_{j+k=N+1}^{\infty}\left|a_{j, k} z^{j}{ }^{k}\right|<\epsilon$.

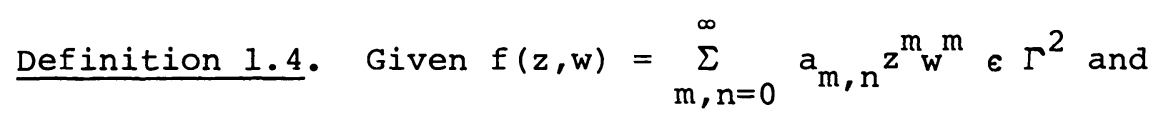
$g(z, w)=\sum_{m, n=0}^{\infty} b_{m, n} z^{m} \in \Gamma^{2}$, define $d(f, q)=$ $\sup \left\{\left|a_{0,0^{-b}} b_{0}\right|,\left|a_{m, n}-b_{m, n}\right|^{1 / m+n: m+n} \geq 1\right\}$.

Theorem 1.5. The space $\left(\Gamma^{2}, d\right)$ is a metric space. Proof. Given $\mathrm{f}, \mathrm{g}$ as in Definition 1.4 , the set $\left\{\left|a_{m, n}-b_{m, n}\right|^{1 / m+n}: m+n \geq 1\right\}$ is a bounded set by Lemma 1.3 , so $d$ is well defined. It is clear that $d(f, g)=0$ if and only if $f=g$ and that $d(f, g)=d(g, f)$. Let $h(z, w)=\sum_{m, n=0}^{\infty} c_{m, n^{2}} z^{m} \in \Gamma^{2}$. Then $d(f, h)=\sup \left\{\left|a_{0,0^{-c}} 0,0\right|,\left|a_{m, n}-b_{m, n}\right|^{1 / m+n}: m+n \geq 1\right\}=$ $\sup \left\{\left|\left(a_{0,0^{-b}}, 0\right)+\left(b_{0,0^{-c_{0}}, 0}\right)\right|,\left|\left(a_{m, n}-b_{m, n}\right)+\left(b_{m, n}-c_{m, n}\right)\right|^{1 / m+n}: m+n \geq 1\right\}$ $\leq \sup \left\{\left|a_{0,0^{-b}} b_{0}\right|+\left|b_{0,0^{-c}} 0,0\right|,\left|a_{m, n}-b_{m, n}\right|^{1 / m+n}+\left|b_{m, n}-c_{m, n}\right|^{1 / m+n}:\right.$ $m+n \geq 1\} \leq \sup \left\{\left|a_{0}, 0^{-b} 0,0\right|,\left|a_{m, n}-b_{m, n}\right|^{1 / m+n}: m+n \geq 1\right\}+\sup$ 
$\left\{\left|b_{0,0^{-c}} 0,0\right|,\left|b_{m, n}-c_{m, n}\right|^{1 / m+n}: m+n \geq 1\right\}=d(f, g)+d(g, h)$. Herice d is a metric on $\Gamma^{2}$.

Section 2. The class of continuous linear functionals on $\Gamma^{2}$.

Definition 2.1. A function $F$ from $\Gamma^{2}$ to $\varnothing$ (complex plane)

is a linear functional if and only if for all $f, g \in \Gamma^{2}, \alpha \in \not{C}, F(f+g)$

$=F(f)+F(g)$ and $F(\alpha f)=\alpha F(f)$.

Definition 2.2. A function $F$ from $\Gamma^{2}$ to $\varnothing$ is said to be continuous at $\mathrm{f} \epsilon \Gamma^{2}$ if and only if for any $\varepsilon>0$ there exists a $\delta>0$ such that if $g \in \Gamma^{2}$ and $d(f, g)<\delta$, then $|F(f)-F(g)|<\epsilon$.

Definition 2.3. A function $F$ from $\Gamma^{2}$ to $\varnothing$ is said to be continuous if and only if it is continuous at each $f \in \Gamma^{2}$.

Lemma 2.4 The series $\sum_{m+n=0}^{\infty} a_{m, n} b_{m, n}$ converges for all sequences $<a_{m, n}>_{m+n=0}^{\infty}$ such that $\lim _{m+n \rightarrow \infty}\left|a_{m, n}\right|^{1 / m+n}=0$ if and only if $<\left|b_{m, n}\right|^{1 / m+n}>_{m+n=1}^{\infty}$ is a bounded sequence.

Proof. Let $\left\langle\left|b_{m, n}\right|^{1 / m+n}>_{m+n=1}^{\infty}\right.$ be a bounded sequence and $<a_{m, n}>_{m+n=0}^{\infty}$ be such that $\lim _{m+n \rightarrow \infty}\left|a_{m, n}\right|^{1 / m+n}=0$. Choose $M>0$ such that $\left|b_{m, n}\right|^{1 / m+n} \leq M$ if $m+n \geq 1$ and then $N \geq 0$ such that $m+n>N \Rightarrow$ $\left|a_{m, n}\right|^{1 / m+n} \leq \frac{1}{2 M} \cdot$ Then if $m+n>N,\left|a_{m, n} b_{m, n}\right| \leq \frac{1}{(2 M)^{m+n}} \cdot M^{m+n}=\frac{1}{2^{m+n}}$. Therefore $\sum_{m+n=N+1}^{\infty}\left|a_{m, n} b_{m, n}\right| \leq \sum_{m+n=N+1}^{\infty} \frac{1}{2^{m+n}}<\infty$. 
Hence the series $\sum_{m+n=0}^{\infty} a_{m, n} b_{m, n}$ converges absolutely, hence it converges.

Conversely suppose for any sequence $<a_{m, n}>_{m+n=0}^{\infty}$ such that $\lim _{m+n \rightarrow \infty}\left|a_{m, n}\right|^{1 / m+n}=0$, the series $\sum_{m+n=0}^{\infty} a_{m, n} b_{m, n}$ converges. If $\left\langle\left|b_{m, n}\right|^{1 / m+n}\right\rangle_{m+n=1}^{\infty}$ is not bounded, for each $k \in z^{+}$there exists an $\left(m_{k}, n_{k}\right)$ such that $\left|b_{m_{k}, n_{k}}\right|^{\frac{1}{m_{k}+n_{k}}}>k$ and $<m_{k}+n_{k}>_{k=1}^{\infty}$ is strictly incfeasing. Choose $a_{m, n}=0$ if $(m, n) \neq\left(m_{k}, n_{k}\right)$, $a_{m_{k}, n_{k}}=k^{\overline{m_{k}+n_{k}}}$. Then $\lim _{m+n \rightarrow \infty}\left|a_{m, n}\right|^{1 / m+n}=\lim _{k} \operatorname{mon}_{k \rightarrow \infty}\left|a_{m_{k}, n_{k}}\right|$ $\frac{1}{m_{k}+n_{k}}=\lim _{k \rightarrow \infty} \frac{1}{k}=0$. But $\left|a_{m_{k}, n_{k}} b_{m_{k}, n_{k}}\right|>1$ for each $k$ so $\sum_{m+n=0}^{\infty} a_{m, n} b_{m, n}$ does not converge. Therefore $\left\langle\left|b_{m, n}\right|^{1 / m+n}>_{m+n=1}^{\infty}\right.$ is bounded. The series $\Sigma a_{m, n} b_{m, n}$ does not converge since the only $\neq 0$ terms are $>1$ and there are an infinite number of them : We now characterize the class of continous linear functionals on $\Gamma^{2}$.

Theorem 2.5. Let $F$ be a function from $\Gamma^{2}$ to the complex plane. Then $F$ is a continous linear functional on $\Gamma^{2}$ if and only if there is a unique sequence $\left\langle b_{m, n}>_{m+n=0}^{\infty}\right.$ such that $\left\langle\left|b_{0,0}\right|,\left|b_{m, n}\right|^{1 / m+n}>_{m+n=1}^{\infty}\right.$ is bounded and such that for all $f(z, w)=\sum_{m+n=0}^{\infty} a_{m, n} z^{m} w^{n} \in \Gamma^{2}$,

$$
F(f)=\sum_{m+n=0}^{\infty} a_{m, n} b_{m, n} \text {. }
$$


Proof. Let $\left\langle\left|b_{0,0}\right|,\left|b_{m, n}\right|^{1 / m+n}>_{m+n=1}^{\infty}\right.$ be a bounded sequence, $M>0$ be such that $\left|b_{0,0}\right|<M,\left|b_{m, n}\right|^{1 / m+n}<M, m+n \geq 1$ and $f(z, w)=\sum_{m+n=0}^{\infty} a_{m, n} z^{m} w^{n} \in \Gamma^{2}$. Then $\lim _{m+n \rightarrow \infty}\left|a_{m, n}\right|^{1 / m+n}=0$ so $\sum_{m+n=0}^{\infty} a_{m, n} b_{m, n}$ converges by Lemma 2.4. Hence we may define a function $F$ from $\Gamma^{2}$ to the complex plane by $F(f)=$ $\sum^{\infty} a_{m, n} b_{m, n}$. It is clear that $F$ is a linear functional. $m+n=0 \quad m, n m, n \cdot$ It is clear that ${ }^{2}$ is a linear functional. Let $\varepsilon>0$ and $f(z, w)=\sum_{m+n=0}^{\infty} a_{m, n} z^{m} w^{n} \in \Gamma^{2}$ be given. We show $m+n=0$
there exists a $\delta>0$ such that if $g \in \Gamma^{2}$ and $d(f, g)<\delta$, then $|F(f)-F(g)|<\epsilon$. Choose $\delta>0$ such that $\delta M<1$ and

$$
\begin{aligned}
\delta M+ & \left(\frac{\delta M}{1-\delta M}\right)^{2}<\epsilon . \text { Then if } g(z, w)=\sum_{m+n=0}^{\infty} C_{m, n} z^{m} w^{n} \in \Gamma^{2} \text { and } \\
d(f, g) & <\epsilon,|F(f)-F(g)|=|F(f-g)|=\left|\sum_{m+n=0}^{\infty}\left(a_{m, n}-C_{m, n}\right) b_{m, n}\right| \\
& \leq\left|a_{0,0}-C_{0,0}\right| M+\sum_{m+n=1}^{\infty}\left|a_{m, n}-C_{m, n}\right| M^{m+n} \\
& \leq \delta M+\sum_{m+n=1}^{\infty}(\delta M)^{m+n} \\
& =\delta M+\sum_{m=1}^{\infty}(\delta M)^{m} \sum_{n=1}^{\infty}(\delta M)^{n} \\
& =\delta M+\left(\frac{\delta M}{1-\delta M}\right)^{2}<\epsilon .
\end{aligned}
$$

Conversely, let $F$ be a continuous linear functional on $\Gamma^{2}$. Let $F\left(z^{m} w^{n}\right)=b_{m, n}$ for all $m+n \geq 0$. Given $f(z, w)=\sum_{m+n=0}^{\infty} a_{m, n} z^{m} w^{n}$, let $f_{N}(z, w)=\sum_{m+n=0}^{N} a_{m, n} z^{m} w^{n}$. Then $d\left(f_{N}, f\right)=\sup \left\{\left|a_{m, n}\right|^{1 / m+n}: m+n\right.$ $>N\} \rightarrow 0$ as $N \rightarrow \infty$ so by the continuity of $F, F\left(f_{N}\right) \rightarrow F(f)$ as $N \rightarrow \infty$. 
But $F\left(f_{N}\right)=\sum_{m+n=0}^{\infty} a_{m, n} b_{m, n}$. Therefore $\lim _{N \rightarrow \infty} \sum_{m+n=0}^{N} a_{m, n} b_{m, n}=F(f)$. Hence $\sum_{m+n=0}^{\infty} a_{m, n} b_{m, n}$ converges and $F(f)=\sum_{m+n=0}^{\infty} a_{m, n} b_{m, n}$ By Lemma 2.4 , the sequence $\left\langle\left|\mathrm{b}_{0,0}\right|^{1 / m+n}{ }_{m+n=1}^{\infty}\right.$ is bounded. Suppose $<C_{m, n}>_{m+n=0}^{\infty}$ is a sequence such that for all $f(z, w)=\sum_{m+n=0}^{\infty} a_{m, n} z^{m} w^{n} \in \Gamma^{2}, F(f)=\sum_{m+n=0}^{\infty} a_{m, n} c_{m, n}$, then for $j, k \in z_{+}, F\left(z^{j} w^{k}\right)=c_{j, k} \cdot$ But $F\left(z^{j}{ }^{k}\right)=b_{j, k}$. Hence $c_{j k}=b_{j, k}$ and the sequence is unique.

Section 3. The class of continuous scalar homomorphisms on $\Gamma^{2}$. Let $f, g \in \Gamma^{2}, \alpha \in \varnothing$ (complex field). Define

$(f+g)(z, w)=f(z, w)+g(z, w),(f \circ g)(z, w)=f(z, w) g(z, w),(\alpha f)$ $(z, w)=\alpha \cdot f(z, w)$. Then $\Gamma^{2}$ becomes a commutative algebra with a unit. In this section we characterize the continuous linear functionals on $\Gamma^{2}$ that preserve multiplication. That is the continuous scalar homomorphisms on $\Gamma^{2}$.

Lemma 3.1. Given $\epsilon>0$ and $(b, c) \epsilon \ell \times \not \subset$, there exists a $\delta>0$ such that if $f, g \in \Gamma^{2}$ and $d(f, g)<\delta$, then $|f(b, c)-g(b, c)|<\epsilon$.

Proof. Given $\epsilon>0$ and $(b, c) \in \not x \not l$, let $R=\max \{|b|,|c|\}$ choose $\delta>0$ such that $\delta R<1$ and $\delta+\left(\frac{\delta R}{1-\delta R}\right)^{2}<\epsilon$. Then if

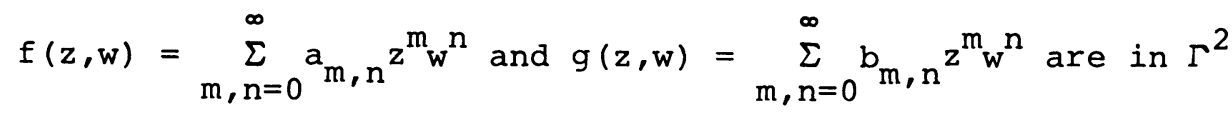
and $d(f, g)<\delta,|f(b, c)-g(b, c)|=\left|\sum_{m+n=0}^{\infty}\left(a_{m, n}-b_{m, n}\right) b^{m} c^{n}\right|$ $\leq\left|a_{0,0^{-b}} 0,0\right|+\sum_{m+n=1}^{\infty}\left|a_{m, n}-b_{m, n}\right| R^{m+n}<\delta+\sum_{m+n=1}^{\infty}(\delta R)^{m+n}=$ 
$\delta+\sum_{m=1}^{\infty}(\delta R)^{m} \sum_{n=1}^{\infty}(\delta R)^{n}=\delta+\left(\frac{\delta R}{1-\delta R}\right)^{2}<\varepsilon$

Theorem 3.2. Let $F$ be a function from $\Gamma^{2}$ to $\not, F \neq 0$.

Then $\mathrm{F}$ is a continuous scalar homorphism on $\Gamma^{2}$ if and only if there exists a unique $(b, c) \in \operatorname{lx} l$ such that for all $f(z, w)=$

$\sum_{m, n=0}^{\infty} a_{m, n} z^{m} w^{n} \in \Gamma^{2}$,

$$
F(f)=f(b, c)
$$

Proof. Let $\mathrm{F}$ be a By Theorem 2.5 , there is a unique sequence $\left\langle b_{m, n}>_{m+n=0}^{\infty}\right.$ such that for all $f(z, w)=\sum_{m, n=0}^{\infty} a_{m, n} z^{m} w^{n} \in \Gamma^{2}, F(f)=\sum_{m+n=0}^{\infty} a_{m, n} b_{m, n}$. For each $m$ and $n, b_{m, n}=F\left(z^{m} w^{n}\right)=F(z)^{m_{F}(w)^{n}}=b_{1,0}^{m} b_{0,1}^{n}$. Therefore $F(f)=\sum_{m+n=0}^{\infty} a_{m, n} b_{1,0}^{m} b_{0,1}^{n}=f\left(b_{1,0} b_{1}\right)$

Conversely, given $(b, c) \in \not{C x} \not \subset$, define a function $F$ from $\Gamma^{2}$ to $\varnothing$ by $F(f)=f(b, c)$. Then $F$ is clearly a Given $\epsilon>0$, let $\delta>0$ be such that if $f, g \in \Gamma^{2}$ and $d(f, g)<\delta$, then $|f(b, c)-g(b, c)|<\epsilon$. Then $|F(f)-F(g)|=|F(f-g)|=$ $|(f-g)(b, c)|=|f(b, c)-g(b, c)|<\epsilon$. Hence $F$ is continuous.

Section 4. The class of bounded linear functionals on $\Gamma^{2}$.

Definition 4.1 . Let $F$ be a linear functional on $\Gamma^{2}$. Then $F$ is said to be bounded if and only if there exists an $M \geq 0$ such 
that for all $f \in \Gamma^{2},|F(f)| \leq \operatorname{Md}(f, 0)$. Here, 0 denotes the function identically zero on $\not{\varnothing x} \not \subset$.

Lemma 4.2. Let $F$ be a linear functional on $\Gamma^{2}$. If $F$ is bounded, $\mathrm{F}$ is continuous but not conversely.

Proof. Let $F$ be a bounded linear functional on $\Gamma^{2}$. Let $f_{0} \epsilon \Gamma^{2} \epsilon>0$ be given and let $M \geq 0$ be such that for all $f \in \Gamma^{2},|F(f)| \leq M d(f, 0)$. Choose $\delta=\epsilon / M+1$. Then if $g \in \Gamma^{2}$ and $d\left(f_{0}, g\right)<\delta,\left|F\left(f_{0}\right)-F(g)\right|=$ $\left|F\left(f_{0}-g\right)\right| \leq M d\left(f_{0}-g, O\right) \leq(m+1) d\left(f_{O}, g\right)<(M+1) \delta<\epsilon$. Therefore $F$ is continuous at $f_{0^{\circ}}$ Hence $F$ is continuous.

For an example of a continuous linear functional that is not bounded, let $b_{m, n}=n$. Then $\left\langle|n|^{1 / m+n}>_{m+n=1}^{\infty}\right.$ is a bounded sequence. Define a function $F$ from $\Gamma^{2}$ to $\not$ by $F\left(\sum_{m, n=0}^{\infty} a_{m, n} z^{m} w^{n}\right)=\sum_{m+n=1}^{\infty} n a_{m, n}$. By Theorem 2.5, $\mathrm{F}$ is a continuous linear functional on $\Gamma^{2}$. If $\mathrm{F}$ is bounded, there exists an $M \geq 0$ such that $\left|\sum_{m+n+1}^{\infty} n a_{m, n}\right| \leq M$ $\sup \left\{\left|a_{0,0}\right|,\left|a_{m, n}\right|^{1 / m+n}: m+n \geq 1\right\}$ for all $<a_{m, n}>_{m+n=0}^{\infty}$ such that $\left|a_{m, n}\right|^{1 / m+n} \rightarrow 0$ as $m+n \rightarrow \infty$. Let $k \in z^{+}, k>\max \{M, 2\}$. Let $a_{0, k}=k, a_{m, n}=0$ if $(m, n)=(0, k)$. Then $\left|a_{m, n}\right|^{1 / m+n} \rightarrow 0$ as $m+n \rightarrow \infty$ since the sequence has only one non-zero term. But 
$\left|\sum_{m+n=1}^{\infty} n a_{m, n}\right|=k^{2}, M \sup \left\{\left|a_{0,0}\right|,\left|a_{m, n}\right|^{1 / m, n}: m+n \geq 1\right\}=$ $M \bullet k^{1 / k}<k \bullet k^{1 / k}<k \bullet k^{1 / k}<k^{2}$, a contradiction. Hence $F$ is not bounded.

Definition 4.3. Let $B$ denote the class of bounded linear functionals on $\Gamma^{2}$. For $F, G \in B, \alpha \in \not \subset, f \in \Gamma^{2}$, define $(F+G)(f)=$ $F(f)=G(f),(\alpha F)(f)=\alpha \cdot F(f),\|F\|=\inf \left\{M \geq 0 \mid\right.$ for all $f \in \Gamma^{2}$, $|F(f)| \leq \operatorname{Md}(f, 0)\}$.

Theorem 4.4. With respect to Definition 4.3, $\mathrm{B}$ is a normed linear space.

Proof. Let $F \in B$. Then $|F(f)| \leq\|F\| d(f, 0)$ for all $F \in \Gamma^{2}$. If not, for some $f_{0} \in \Gamma^{2},\left|F\left(f_{0}\right)\right|>\|F\| d\left(f_{0,0}\right)$. Then $d\left(f_{0}, 0\right) \neq 0$ so choose $\epsilon>0$ such that $\left|F\left(f_{0}\right)\right|=\|F\| d\left(f_{0}, 0\right)+\epsilon d\left(f_{0}, 0\right)$. By Definition of ||$F||$, there exists an $M \geq 0$ such that $|F(f)| \leq M d$ $(f, 0)$ for all $f \in \Gamma^{2}$ and $\|F\|+\epsilon>M$. Then $d\left(f_{0}, 0\right)(\|F\|+\epsilon)$ $=\left|F\left(f_{0}\right)\right| \leq M d\left(f_{o}, 0\right)$. Hence ||$F||+\epsilon \leq M$, a contradiction. Therefore $|F(f)| \leq\|F\| d(f, 0)$ for all $f \in \Gamma^{2}$ and $\|F\|$ is the smallest number to satisfy this inequality for all $f \in \Gamma^{2}$.

For $F, G \in B, \alpha \in \not{C}, F+G$ and $\alpha F$ are clearly linear functionals on $\Gamma^{2}$. For $f \in \Gamma^{2},|(F+G)(f)|=|F(f)+G(f)| \leq|F(f)|+|G(f)| \leq$ $\|F\| d(f, 0)+\|G\| d(f, 0)=(\|F\|+\|G\|) d(f, 0)$. Hence $F+G \in B$ and ||$F+G|| \leq|| F||+|| G||$. Also $|(\alpha F)(f)|=|\alpha \cdot F(f)|=$ $|\alpha||F(f)| \leq|\alpha|\|F\| \quad d(f, 0)$. Hence $\alpha F \in B$ and ||$\alpha F|| \leq|\alpha|$ $\|F\|$. Suppose it is possible to have ||$\alpha F\|<|\alpha|\| F \|$. Choose 
$\epsilon>0$ such that ||$\alpha F||+\epsilon|\alpha||| F||=|\alpha||| F||$. Then for all

$f \in \Gamma^{2},|\alpha||F(f)|=|(\alpha F)(f)| \leq|| \alpha F|| \alpha(f, 0)=(1-\epsilon)|\alpha|$

||$F|| d(f, 0)$. Therefore $|F(f)| \leq(1-\epsilon)|| F|| d(f, 0), a$

contradiction. Hence ||$\alpha F||=|\alpha||| F||$.

It is clear that $\|\cdot\|$ evaluated at the zero linear

functional on $\Gamma^{2}$ is 0 and if ||$F||=0$ then $|F(f)|=0$ for all

$f \in \Gamma^{2}$, hence $F \equiv 0$. Also the remaining properties required for $B$ to be a normed linear space follow trivially. Hence $B$ is a normed linear space with respect to Definition 4.3 .

Theorem 4.5. Let $F$ be a function from $\Gamma^{2}$ to $\not$. Then $F \in$ B if and only if there exists unique $(a, b, c,) \in \mathbb{\ell} \times \mathbb{\ell} \not$ such that for $\operatorname{all} f(z, w)=\sum_{m, n=0}^{\infty} a_{m, n^{m}} z^{m} \in \Gamma^{2}$,

$$
F(f)=a_{0,0} a+a_{1,0} b+a_{0,1} c
$$

Also, ||$F||=|a|+|b|+|c|$.

Proof. Let $F \in B$. Then $F$ is continuous so there exist a unique sequence $\left\langle b_{m, n}>_{n+n=0}^{\infty}\right.$ such that $F\left(\sum_{m, n=0}^{\infty} a_{m, n} z^{m} w^{n}\right)=\sum_{m+n=0}^{\infty} a_{m, n} b_{m, n}$ and $\left|\sum_{m+n=0}^{\infty} a_{m, n} b_{m, n}\right| \leq|| F|| \sup \left\{\left|a_{0,0}\right|,\left|a_{m, n}\right|^{1 / m+n}: m+n \geq 1\right.$ for all sequences $<a_{m, n}>_{m+n=0}^{\infty}$ such that $\left|a_{m, n}\right|^{1 / m+n} \rightarrow 0$ as $m+n \rightarrow \infty$. Suppose $b_{k, j} \neq 0$ for some $(k, j)$ with $k+j \geq 2$. Choose $a_{m, n}=0$ if $(m, n) \neq(k, j)$ and choose $a_{k, j}$ such that ||$F||>$ $\left|a_{k, j}\right|^{1-\frac{1}{k+j}} \cdot\left|b_{k, j}\right|$. Then $\left|a_{m, n}\right|^{1 / m+n} \rightarrow 0$ as $m+n \rightarrow \infty$, 
$\left|\sum_{m+n=0}^{\infty} a_{m, n} b_{m, n}\right| \equiv\left|a_{k \cdot j} b_{k, j}\right| \leq|| F||\left|a_{k, j}\right|^{\frac{1}{k+j}}$ Therefore $\left|a_{k, j}\right| 1-\frac{1}{k+j} \cdot\left|b_{k, j}\right| \leq \| F||$, a contradiction. Hence $b_{k, j}=0$ if $k+j \geq 2$. Hence $F\left(\sum_{m, n=0}^{\infty} a_{m, n} z^{m} w^{n}\right)=a_{0,0} b_{0,0}+a_{1,0} b_{1,0}+$ $a_{0,1} b_{0,1}$. Also $\left|F\left(\sum_{m, n=0}^{\infty} a_{m, n} z^{m} w^{n}\right)\right| \leq\left|a_{0,0}\right|\left|b_{0,0}\right|+$ $\left|a_{1,0}\right|\left|b_{1,0}\right|+\left|a_{0,1}\right|\left|b_{0,1}\right| \leq\left(\left|b_{0,0}\right|+\left|b_{1,0}\right|+\left|b_{0,1}\right|\right) d(f, 0)$. Therefore ||$F|| \leq\left|b_{0,0}\right|+\left|b_{1,0}\right|+\left|b_{0,1}\right|$. To show equality here, it sufficies to show there exists an $f_{0} \in \Gamma^{2}$ such that $\left|F\left(f_{0}\right)\right|=\left(\left|b_{0,0}\right|+\left|b_{1,0}\right|+\left|b_{0,1}\right|\right) d\left(f_{0,0}\right)$. If $b_{0,0}=$ $\left|b_{0,0}\right| e^{i \theta} 1, b_{1,0}=\left|b_{1,0}\right| e^{i \theta} 2, b_{0,1}=\left|b_{0,1}\right| e^{i \theta_{3}}$, choose $f_{0}(z, w)=e^{-i \theta_{1}}+e^{-i \theta_{2}} z+e^{-i \theta_{3}} w$. Then $\left|F\left(f_{0}\right)\right|=$ $\left|b_{0,0}\right|+\left|b_{1,0}\right|+\left|b_{0,1}\right|=\left(\left|b_{0,0}\right|+\left|b_{1,0}\right|+\left|b_{0,1}\right|\right) d\left(f_{0}, 0\right)$. Conversely, given $(a, b, c) \in \mathbb{\ell} \times \mathbb{\ell} \not \mathbb{l}$, define a function $\mathrm{F}$ from $\Gamma^{2}$ to $\not$ by $F(f)=a_{0,0} a+a_{1,0} b+a_{0,1} c, f(z, w)$ $\sum_{m, n=0}^{\infty} a_{m}, n^{m^{m}} w^{n} \in \Gamma^{2}$. By Theorem 2.5, F is a continuous linear functional on $\Gamma^{2}$. Since $|F(f)| \leq\left|a_{0,0}\right||a|+\left|a_{1,0}\right||b|+$ $\left|a_{0,1}\right||c| \leq(|a|+|b|+|c|) d(f, 0), F \in B$.

Corollary 4.6. With respect to Definition 4.3, B is a Banach space. 
Proof. Let $\left\langle F_{n}>_{n=1}^{\infty}\right.$ be a Cauchy sequence in $B, F_{n}$ corresponding to $\left(a_{n}, b_{n}, c_{n}\right)$. Then for any $\epsilon>0$, there exists an $N=N(\varepsilon)$ such that $m, n>N(\epsilon) \Rightarrow|| F_{n}-F_{m}||<\epsilon$. That is $\left|a_{n}-a_{m}\right|+\left|b_{n}-b_{m}\right|+\left|a_{n}-c_{m}\right|$ $<\epsilon$. Hence each of $\left\langle a_{n}\right\rangle,\left\langle b_{n}\right\rangle,\left\langle c_{n}\right\rangle$ is a Cauchy sequence. Let $a_{n} \rightarrow a, b_{n} \rightarrow b, c_{n} \rightarrow c$ as $n \rightarrow \infty$. Define a function $F$ from $\Gamma^{2}$ to $\varnothing$ by $F\left(\sum_{m, n=0}^{\infty} a_{m, n^{2}} z^{m}\right)=a_{0,0} a+a_{1,0} b+a_{0,1} c$. By Theorem 4.5, F $\in$ B. Given $\epsilon>0$, there exists an $N=N(\epsilon)$ such that $\left|a_{m}-a\right|+\left|b_{m}-b_{n}\right|$ $+\left|c_{m}-c_{n}\right|<\epsilon / 2$ if $m, n>N(\epsilon)$. Let $m \rightarrow \infty$ to get $\left|a-a_{n}\right|+\left|b-b_{n}\right|$ $+\left|c-c_{n}\right| \leq \epsilon / 2$ if $n>N(\epsilon)$. Hence given $\epsilon>0$, there exists an $N=N(\epsilon)$ such that if $n>N(\epsilon),\left|a-a_{n}\right|+\left|b-b_{n}\right|+\left|c-c_{n}\right|<\epsilon$. That is $\| F_{n}-F||<\epsilon$. Therefore $B$ is a Banach space. If $\left(a_{1}, b_{1}, c_{1}\right)$ and $\left(a_{2}, b_{2}, c_{2}\right)$ are in $\not x \not \times \not \subset$ and $\alpha \in \not l$, it is clear that if addition and scalar multiplication are defined by $\left(a_{1}, b_{1}, c_{1}\right)+\left(a_{2}, b_{2}, c_{2}\right)=\left(a_{1}+a_{2}, b_{1}+b_{2}, c_{1}+c_{2}\right), \alpha\left(a_{1}, b_{1}, c_{1}\right)=$ $\left(\alpha a_{1}, \alpha b_{1}, \alpha c_{1}\right)$, that $\ell \times \ell \times \ell$ is a vector space over $\ell$. Also if ||$\cdot||$ is defined by $\|(a, b, c)||=|a|+|b|+|c|,\| \cdot||$ is a norm

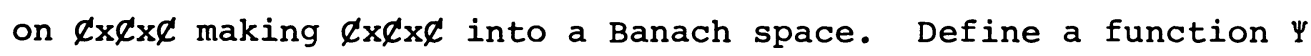
from $B$ to $\not x \not x \not l$ as follows: For $F \in B$, let $(a, b, c)$ be the unique element of $\ell \times \not \times \varnothing$ such that $F(f)=a_{0,0} a+a_{1} 0^{b}+a_{0,1} c$ for all $f(z, w)=\sum_{m, n=0}^{\infty} a_{m, n^{2}} z^{m} \in \Gamma^{2}$. Let $\Psi(F)=(a, b, c)$. The following theorem is straightforward to prove so the proof is omitted 
Theorem 3.4.7. The spaces B and $\operatorname{Ex} \notin$ are isometrically isomorphic Banach spaces.

\section{REFERENCES}

1. Copson, E. T. Theory of Functions of a Complex Variable, Oxford Clarendon Press, 1935.

2. Funks, B. A. Theory of Analytic Functions of Several Complex Variables, American Mathematical Society, Providence, 1963.

ABSTRACT. Three classes of linear functionals on the space of entire functions of two variables are characterized. Several results are proved.

ACKNOWLEDGMENT. This work was supported by a 1976 Marshall University Summer Research Grant.

KEY WORDS AND PHRASES. Space of entire functions, Metric space, continuous linear functional and continuous scalar homorphism.

AMS (MOS) SUBJECT CLASSIFICATIONS (1970). 30A66, $46 E 15$. 


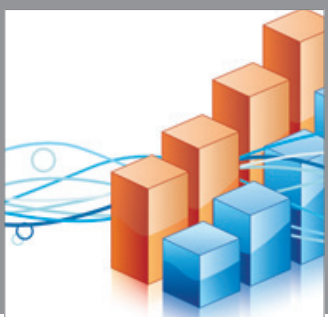

Advances in

Operations Research

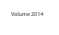

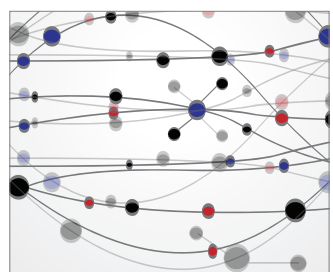

\section{The Scientific} World Journal
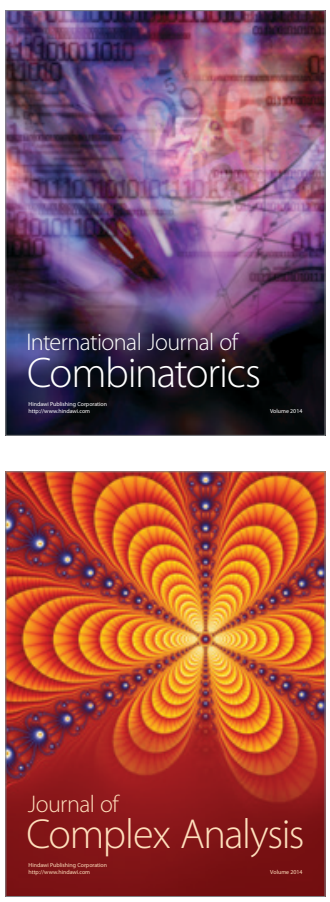

International Journal of

Mathematics and

Mathematical

Sciences
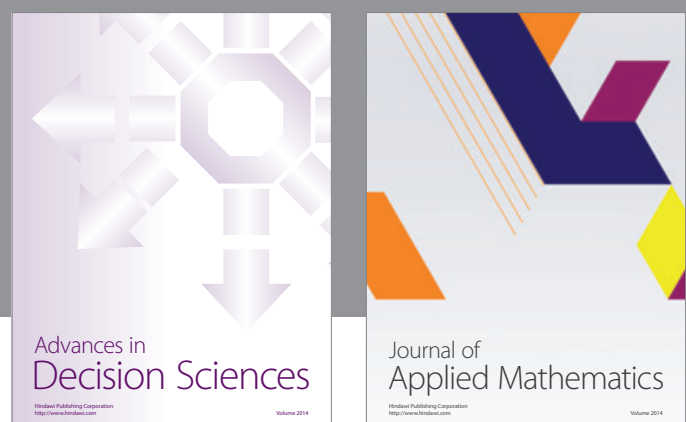

Journal of

Applied Mathematics
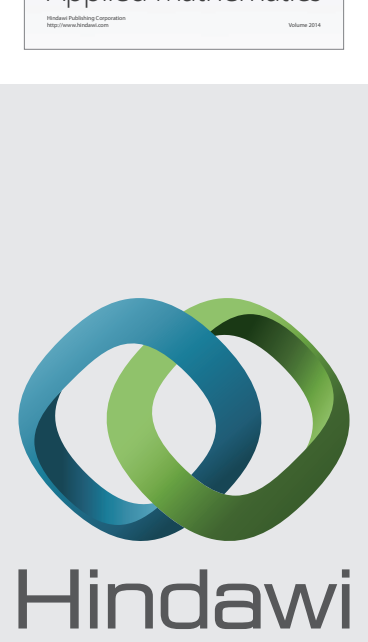

Submit your manuscripts at http://www.hindawi.com
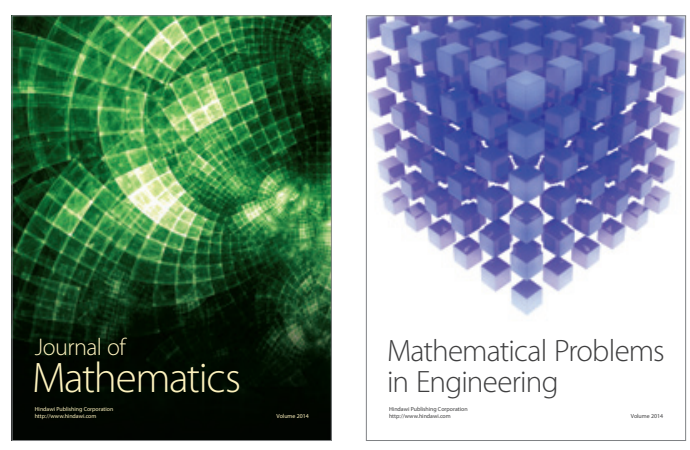

Mathematical Problems in Engineering
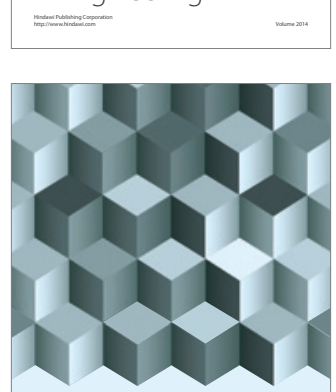

Journal of

Function Spaces
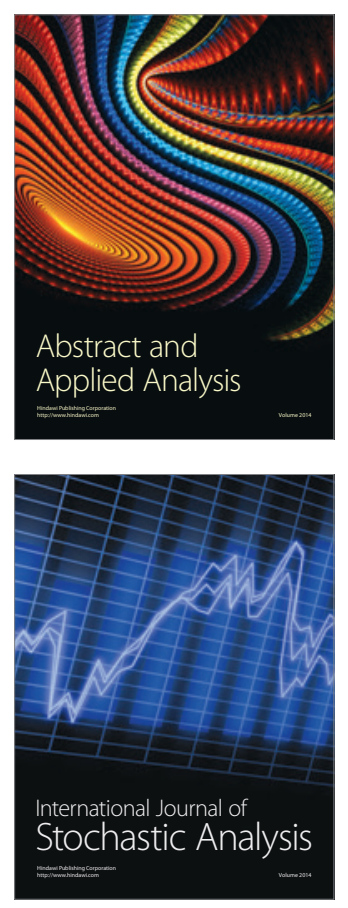

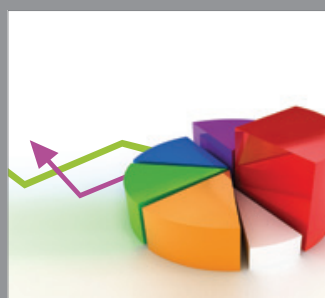

ournal of

Probability and Statistics

Promensencen
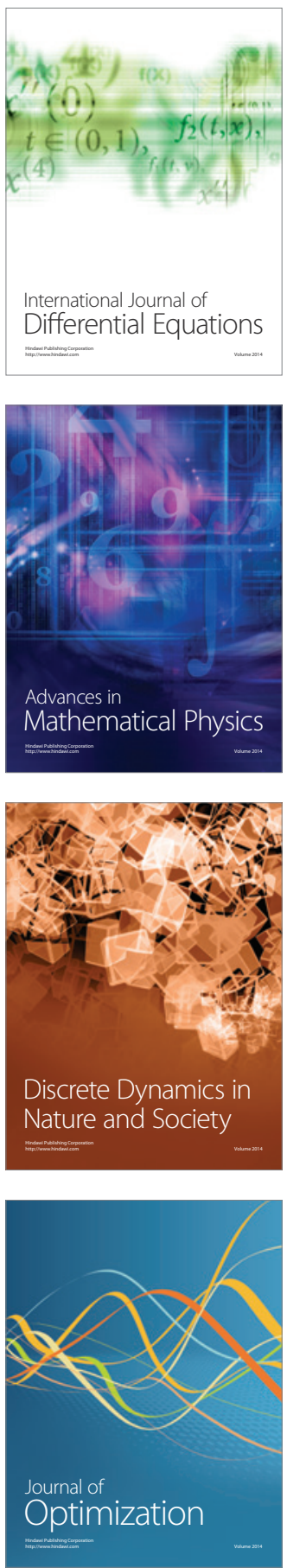